\title{
Anticoagulation versus Antiplatelet Therapy after Ischemic Stroke in the Patients with Atrial Fibrillation and Cerebral Microbleeds
}

\author{
Kang-Ho Choi, ${ }^{\text {a,b,* }}$ Ja-Hae Kim, ${ }^{\mathrm{c}, *}$ Changho Lee, ${ }^{\mathrm{d}}$ Jae-Myung Kim, ${ }^{\mathrm{a}, \mathrm{b}}$ Kyung-Wook Kang, ${ }^{\mathrm{a}, \mathrm{b}}$ \\ Joon-Tae Kim, ${ }^{a}$ Seong-Min Choi, ${ }^{a}$ Man-Seok Park, ${ }^{a}$ Ki-Hyun Cho ${ }^{a}$ \\ aDepartment of Neurology, Chonnam National University Hospital, Chonnam National University Medical School, Gwangju, Korea \\ 'Department of Neurology, Chonnam National University Hwasun Hospital, Hwasun, Korea \\ 'Molecular Imaging Center, Department of Nuclear Medicine, Chonnam National University Hospital, Chonnam National University Medical \\ School, Gwangju, Korea \\ 'Molecular Imaging Center, Department of Nuclear Medicine, Chonnam National University Hwasun Hospital, Chonnam National University \\ Medical School, Hwasun, Korea
}

*These authors contributed equally to the manuscript as first author.

\section{Dear Sir:}

Recent studies, including our previous investigation, indicate that cerebral microbleeds (CMBs) are significantly associated with a higher risk of hemorrhagic stroke among patients taking oral anticoagulants (OACs). ${ }^{1,2}$ Physicians, therefore, are confronted with a dilemma regarding the proper administration of antithrombotic therapy to patients with both atrial fibrillation (AF) and CMB due to the patients' competing ischemic and hemorrhagic conditions. ${ }^{3}$ This dilemma may be especially pronounced in patients with severe CMB patterns (multiple or lobar CMBs). ${ }^{3}$ Nevertheless, no study had directly compared the effectiveness of administering guideline-recommended OACs with that of antiplatelet therapy, which is used in a large portion of patients with AF in real-world practice, for preventing recurrent stroke in patients with both $\mathrm{AF}$ and $\mathrm{CMB}^{4}{ }^{4}$ Therefore, we investigated the efficacy and safety of OACs versus those of antiplatelet therapy in reducing the future risk of stroke in these patients.

This study is a retrospective analysis of a prospective registry. The primary outcome measurement was the incidence of recurrent stroke (ischemic or hemorrhagic) at any time during the final 2 years of follow-up. We used propensity scores and stabilized inverse probability of treatment weighting (IPTW) to balance possible confounders. Detailed methodical descriptions are provided in the Supplementary Methods. This study included 483 patients with CMB and acute ischemic stroke associated with AF (Supplementary Figure 1). The clinical and biochemical characteristics of the patients before and after IPTW are shown in Supplementary Table 1. Compared with those in the OAC group, the patients in the antiplatelet group were more likely to have severe initial symptoms, cerebral atherosclerosis, and a history of diabetes mellitus. Absolute standardized differences were balanced after propensity score matching (Supplementary Figure 2).

We found that 51 patients experienced a stroke during follow-up. Patients treated with OACs had a significantly lower risk of future stroke (hazard ratio [HR], 0.42; 95\% confidence interval [Cl], 0.23 to 0.76; $P=0.004$ ) (Figure 1 and Supplementary Table 2) than did the patients who were treated with antiplatelet therapy. In particular, the non-vitamin K antagonist OACs group had a significantly lower risk of recurrent stroke than did the antiplatelet group $(\mathrm{HR}, 0.22 ; 95 \% \mathrm{Cl}, 0.06$ to 0.74 ; $P=0.014$ ) (Supplementary Table 2). Regarding key secondary outcomes, the OAC group had a significantly lower rate of recurrent ischemic stroke, all-cause death, and major adverse cardiovascular events relative to the antiplatelet group. By contrast, the rates of intracerebral hemorrhage were similar 
Stroke

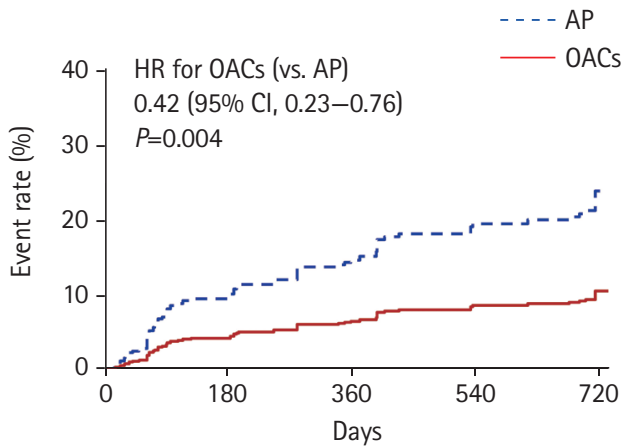

No. at risk

$\begin{array}{rrrrrr}\text { AP } & 479 & 318 & 251 & 202 & 186 \\ \text { OACs } & 483 & 365 & 298 & 235 & 210\end{array}$

Recurrent ischemic stroke - - - AP

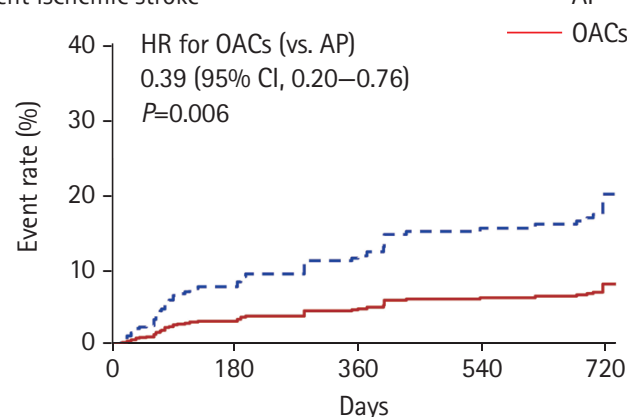

No. at risk

$\begin{array}{rlllll}\text { AP } & 479 & 318 & 251 & 202 & 186 \\ \text { OACs } & 483 & 365 & 298 & 235 & 210\end{array}$

A

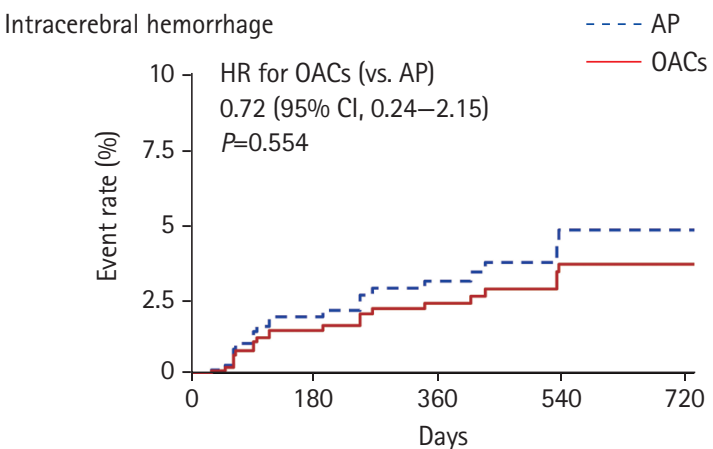

No. at risk

$\begin{array}{rrrrrr}\text { AP } & 479 & 318 & 251 & 202 & 194 \\ \text { OACs } & 483 & 373 & 305 & 240 & 218\end{array}$

Major adverse cardiovascular events - - - AP

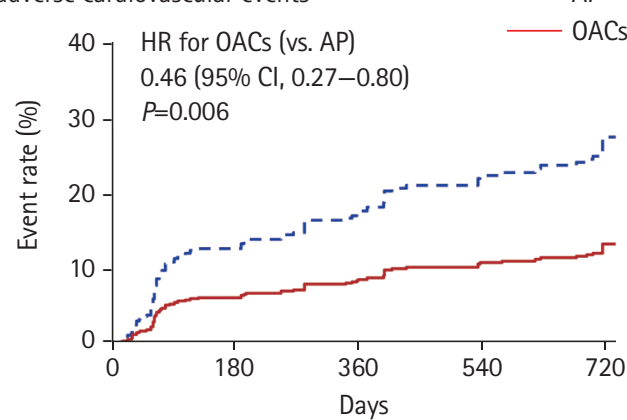

No. at risk

$\begin{array}{rrrrrr}\text { AP } & 479 & 318 & 251 & 202 & 186 \\ \text { OACs } & 483 & 365 & 298 & 235 & 207\end{array}$

C

Figure 1. Incidence rates of stroke (A) and secondary outcomes (B-D) among patients receiving antithrombotic therapy. HR, hazards ratio; OAC, oral anticoagulant; $\mathrm{AP}$, antiplatelet; $\mathrm{Cl}$, confidence interval.

between the antiplatelet and OAC groups (Figure 1 and Supplementary Table 2).

Among the patients with exactly one $\mathrm{CMB}$, the rate of stroke was lower in the OAC group than in the antiplatelet group; however, this difference was nonsignificant $(\mathrm{HR}, 0.43 ; 95 \% \mathrm{Cl}$, 0.13 to 1.28; $P=0.154$ ) (Figure 2 and Supplementary Table 2). By contrast, OACs were significantly associated with a reduced risk of stroke in patients with multiple CMBs (HR, 0.38; 95\% $\mathrm{Cl}, 0.19$ to $0.75 ; P=0.005)$ compared to antiplatelet therapy. Among patients with strictly lobar $C M B$, the risk of stroke was also significantly lower in the OAC group than in the antiplatelet group ( $\mathrm{HR}, 0.36 ; 95 \% \mathrm{Cl}, 0.17$ to $0.75 ; P=0.006)$. However, there was no significant difference in the risk of stroke between the $O A C$ and antiplatelet groups among patients with deep/mixed CMB (Figure 2 and Supplementary Table 2).

Our study suggests that the administration of OACs to isch- emic stroke patients with $\mathrm{AF}$ and $\mathrm{CMB}$ is significantly associated with a lower risk of future stroke than antiplatelet therapy. Despite the common concern that OACs may cause intracerebral hemorrhage in patients with severe CMB patterns, our study shows that patients with multiple or lobar CMBs may benefit more from OACs than antiplatelet therapy. The higher risk of ischemic stroke than hemorrhagic stroke among patients with CMB may account for this finding $i^{1,5}$ the crude incidence of ischemic stroke was more than twofold that of hemorrhagic stroke in our population, even among patients with severe CMB patterns. As the absolute risk of ischemic stroke in patients with $\mathrm{CMBs}$ can increase as the CMB burden increas$\mathrm{es}_{1}{ }^{6}$ choosing to administer OACs rather than antiplatelet therapy would be beneficial to patients with multiple CMBs. The only study to date comparing the effects of OACs with those of antiplatelet therapy in ischemic stroke patients with $\mathrm{AF}$ and 


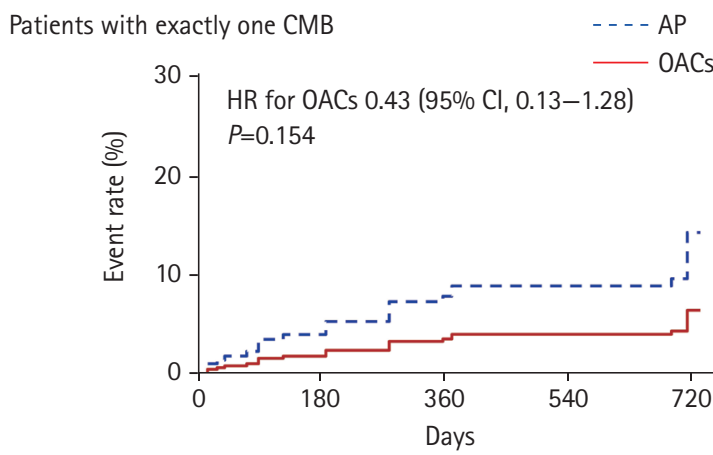

No. at risk

$\begin{array}{rrrrrr}\text { AP } & 218 & 169 & 141 & 120 & 108 \\ \text { OACs } & 215 & 165 & 138 & 108 & 104\end{array}$

Patients with multiple $\mathrm{CMBs}(\geq 2)$

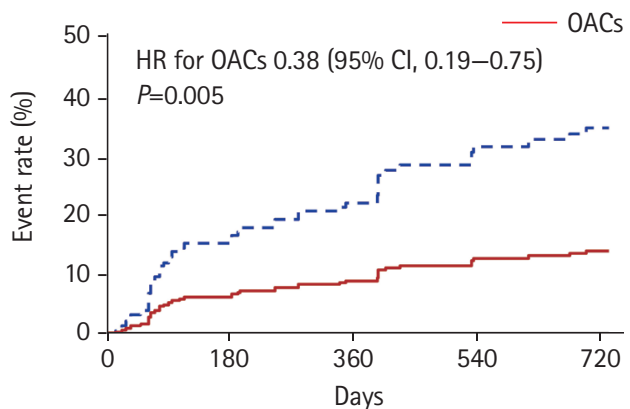

No. at risk

$\begin{array}{rrrrrr}\text { AP } & 261 & 149 & 110 & 82 & 78 \\ \text { OACs } & 268 & 200 & 160 & 127 & 106\end{array}$

A

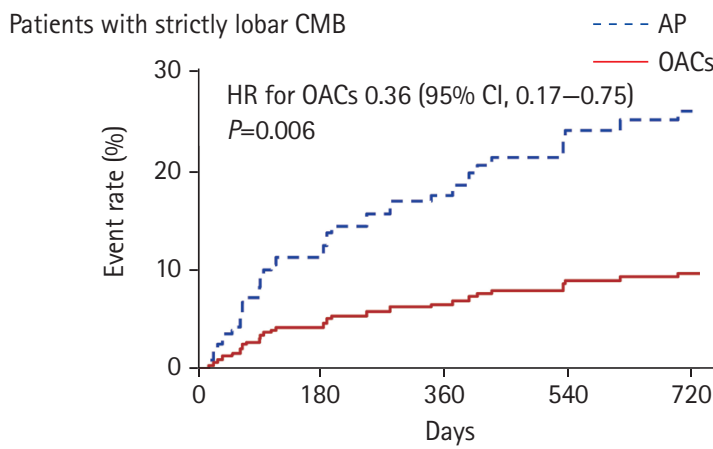

No. at risk

$\begin{array}{rrrrrr}\text { AP } & 271 & 183 & 132 & 104 & 97 \\ \text { OACs } & 275 & 206 & 166 & 129 & 121\end{array}$

Patients with deep/mixed CMB

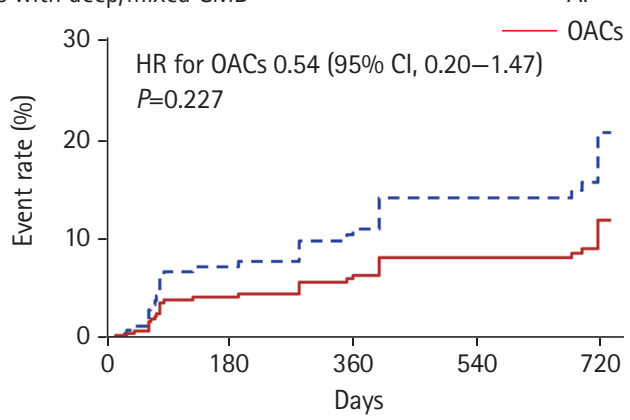

No. at risk

$\begin{array}{rrrrrr}\text { AP } & 208 & 135 & 119 & 98 & 89 \\ \text { OACs } & 208 & 159 & 132 & 106 & 89\end{array}$

C D

Figure 2. The effects of anticoagulation versus that of antiplatelet therapy on stroke risk according to the burden $(A, B)$ and location $(C, D)$ of cerebral microbleed (CMB). AP, antiplatelet; $\mathrm{HR}$, hazards ratio; $\mathrm{OAC}$, oral anticoagulant; $\mathrm{Cl}$, confidence interval.

$\mathrm{CMB}$ estimated that patients with greater $\mathrm{CMB}$ counts might receive a greater benefit from OACs than from aspirin therapy. ${ }^{5}$ However, the estimates of the study were calculated indirectly from the data reported by previous studies. Our real-world study supports previous estimates concerning the benefit of OACs compared to antiplatelet therapy in the prevention of future stroke among ischemic stroke patients with $\mathrm{AF}$ and $\mathrm{CMB}$.

Our study is subject to limitations. As an observational, retrospective analysis of a relatively small sample, this investigation has methodological shortcoming. In addition, our data are prone to the influences of potential selection bias and unmeasured confounders. However, to reduce the risk of confounding, we used propensity score analyses.

In conclusion, our results indicate that for the secondary prevention of stroke in ischemic stroke patients with $\mathrm{AF}$ and $C M B$, the clinical benefits of guideline-recommended OACs can outweigh the risks in comparison to antiplatelet therapy. The net benefits of OAC therapy may be particularly pronounced in patients with multiple or lobar CMBs. Further studies are required to better characterize and tailor optimal antithrombotic treatment for ischemic stroke patients with $\mathrm{AF}$ and $\mathrm{CMB}$.

\section{Supplementary materials}

Supplementary materials related to this article can be found online at https://doi.org/10.5853/jos.2020.04588.

\section{References}

1. Wilson D, Ambler G, Shakeshaft C, Brown MM, Charidimou A, Al-Shahi Salman R, et al. Cerebral microbleeds 
and intracranial haemorrhage risk in patients anticoagulated for atrial fibrillation after acute ischaemic stroke or transient ischaemic attack (CROMIS-2): a multicentre observational cohort study. Lancet Neurol 2018;17:539-547.

2. Choi KH, Kim JH, Lee C, Kim JM, Kang KW, Kim JT, et al. Microbleeds and outcome in patients with acute ischemic stroke and atrial fibrillation taking anticoagulants. Stroke 2020;51:3514-3522.

3. Selim M, Diener HC. Atrial fibrillation and microbleeds. Stroke 2017:48:2660-2664.

4. Xian Y, O'Brien EC, Liang L, Xu H, Schwamm LH, Fonarow $\mathrm{GC}$, et al. Association of preceding antithrombotic treatment with acute ischemic stroke severity and in-hospital outcomes among patients with atrial fibrillation. JAMA 2017;317:1057-1067.

5. Shoamanesh A, Charidimou A, Sharma M, Hart RG. Should patients with ischemic stroke or transient ischemic attack with atrial fibrillation and microbleeds be anticoagulated? Stroke 2017;48:3408-3412.
6. Wilson D, Charidimou A, Ambler G, Fox ZV, Gregoire S, Rayson $\mathrm{P}$, et al. Recurrent stroke risk and cerebral microbleed burden in ischemic stroke and TIA: a meta-analysis. Neurology 2016;87:1501-1510.

Correspondence: Kang-Ho Choi

Department of Neurology, Chonnam National University Hospital, Chonnam National University Medical School, 42 Jebong-ro, Dong-gu, Gwangju 61469, Korea

Tel: $+82-62-220-6137$

Fax: +82-62-228-3461

E-mail: ckhchoikang@chonnam.ac.kr

https://orcid.org/0000-0001-8851-2104

Received: November 11, 2020

Revised: December 30, 2020

Accepted: March 14, 2021

This work was supported by a National Research Foundation of Korea grant funded by the Korean Government (NRF-2019M3A9E8020261). This study was supported by a grant (HCRI20006) from Chonnam National University Hwasun Hospital Institute for Biomedical Science.

The authors have no financial conflicts of interest. 


\section{Supplementary methods}

\section{Subjects and study design}

This study is a retrospective analysis of data deposited in the prospective registry of our government-initiated comprehensive stroke center between January 2010 and January 2018. Patients underwent brain magnetic resonance imaging (MRI) scans, including T2-weighted gradient echo imaging, for the evaluation of cerebral microbleed (CMB) immediately upon admission to the emergency room. Patients were enrolled in the study if they met the following inclusion criteria: (1) had acute ischemic stroke associated with atrial fibrillation and (2) had CMB on brain MRI. We excluded patients if they (1) died during hospitalization; (2) could not be treated with antithrombotic therapy due to contraindications; (3) were treated with a combination of oral anticoagulants (OAC) and antiplatelet at discharge; or (4) whose antithrombotic treatment changed during follow-up period (from OAC to antiplatelet or vice versa) (Supplementary Figure 1).

The patients were divided into groups according to their prescribed antithrombotic therapies, which were determined by their physicians after the index stroke. We allowed the use of any type of antithrombotic medication (antiplatelets, vitamin $\mathrm{K}$ antagonist, or non-vitamin $\mathrm{K}$ antagonist oral anticoagulants). Two blinded neuroimaging specialists identified the number and location of CMBs. The CMB burden was classified as either exactly one CMB or as multiple CMBs ( $\geq 2 \mathrm{CMBs}$ ). The location of CMBs was defined as strictly lobar or deep/mixed. The infratentorial location of CMB was considered deep CMB. The assessment of $\mathrm{CMB}$ number and location showed excellent inter-rater agreement $(K=0.917)$. Baseline characteristics, underlying stroke risk factors, and laboratory data were collected from all subjects.

\section{Outcome measurements}

The primary outcome measurement was the incidence of recurrent stroke (ischemic or hemorrhagic) at any time before the end of the 2-year follow-up. Subjects were grouped ac- cording to their antithrombotic therapy. Key secondary outcomes included the incidence of recurrent ischemic stroke, intracerebral hemorrhage, all-cause death, and major adverse cardiovascular events (a composite of stroke, acute myocardial infarction, or vascular death) over the following 2 years. We prospectively obtained clinical outcomes from all patients during hospitalization, routine clinic visits, or via telephone interviews with patients or their caregivers.

\section{Statistical analysis}

We compared the differences between groups using Student's t-tests or Kruskal-Wallis tests for continuous variables. We used chi-square tests or Fisher's exact tests for categorical variables. We used propensity scores and stabilized inverse probability of treatment weighting (IPTW) to balance possible confounders. An absolute standardized difference was calculated before and after IPTW; a value of $<0.1$ was considered as a low imbalance between the groups. The event rates of clinical outcome measures were estimated using the weighted Kaplan-Meier method and compared between the groups using the IPTW log-rank test. The weighted Cox proportional hazard regression was used to calculate the hazard ratios and 95\% confidence intervals for primary and secondary outcomes, according to the antithrombotic medication received. A two-sided $P$-value of $<0.05$ was considered to indicate statistical significance. Statistical analyses were performed using SPSS version 25.0 (IBM Corp., Armonk, NY, USA) and R software version 3.3.1 (R Foundation, Vienna, Austria).

\section{Ethics statement and data availability}

An Institutional Review Board of Chonnam National University Hospital approved this study. Written informed consent was obtained from all patients or their legal representatives prior to their enrollment in the prospective stroke registry. All the clinical and laboratory investigations described in this study were conducted according to the principles outlined in the Declaration of Helsinki. All supporting data within the article are available upon reasonable request from a qualified investigator. 
Supplementary Table 1. Baseline characteristics according to antiplatelet treatment before and after IPTW

\begin{tabular}{|c|c|c|c|c|c|c|c|c|}
\hline \multirow{2}{*}{ Characteristic } & \multicolumn{4}{|c|}{ Before IPTW } & \multicolumn{4}{|c|}{ After IPTW } \\
\hline & Antiplatelet $(n=130)$ & OACs $(n=353)$ & Std diff & $P$ & Antiplatelet $(n=479)$ & OACs $(n=483)$ & Std diff & $P$ \\
\hline \multicolumn{9}{|l|}{ Demographics } \\
\hline Age in years & $73.7 \pm 9.9$ & $73.1 \pm 8.9$ & 0.067 & 0.506 & $73.2 \pm 10.4$ & $73.3 \pm 8.9$ & 0.005 & 0.939 \\
\hline Male sex & $66(50.8)$ & $168(47.6)$ & 0.063 & 0.605 & $235(49.2)$ & $233(468)$ & 0.018 & 0.825 \\
\hline \multicolumn{9}{|l|}{ Comorbidities } \\
\hline Hypertension & $92(70.8)$ & $234(66.3)$ & 0.096 & 0.411 & $326(68.1)$ & $326(67.5)$ & 0.015 & 0.906 \\
\hline Diabetes mellitus & $42(32.3)$ & 75 (21.2) & 0.251 & 0.017 & $116(24.3)$ & $116(24.0)$ & 0.005 & 0.988 \\
\hline Dyslipidemia & $51(39.2)$ & $143(40.5)$ & 0.026 & 0.881 & $182(38.1)$ & $193(40.0)$ & 0.038 & 0.595 \\
\hline Current smoking & $17(13.1)$ & 49 (13.9) & 0.023 & 0.937 & $72(15.1)$ & $66(13.7)$ & 0.042 & 0.599 \\
\hline Coronary heart disease & $11(8.5)$ & $22(6.2)$ & 0.085 & 0.511 & $29(6.1)$ & $32(6.6)$ & 0.020 & 0.817 \\
\hline Prior stroke or TIA & $40(30.8)$ & $109(30.9)$ & 0.002 & 1.000 & $141(29.5)$ & $148(30.6)$ & 0.024 & 0.752 \\
\hline \multicolumn{9}{|l|}{ Laboratory values } \\
\hline Total cholesterol (mg/dL) & $161.3 \pm 39.7$ & $163.3 \pm 39.5$ & 0.050 & 0.625 & $163.6 \pm 40.3$ & $162.9 \pm 38.7$ & 0.017 & 0.787 \\
\hline $\mathrm{LDL}-\mathrm{C}(\mathrm{mg} / \mathrm{dL})$ & $100.7 \pm 34.6$ & $104.0 \pm 33.5$ & 0.097 & 0.342 & $103.1 \pm 35.6$ & $103.1 \pm 32.9$ & 0.001 & 0.992 \\
\hline Triglyceride (mg/dL) & $88.7 \pm 47.9$ & $89.3 \pm 47.6$ & 0.014 & 0.890 & $87.8 \pm 47.8$ & $89.2 \pm 47.6$ & 0.028 & 0.660 \\
\hline $\mathrm{HDL}-\mathrm{C}(\mathrm{mg} / \mathrm{dL})$ & $45.5 \pm 12.6$ & $45.1 \pm 0.7$ & 0.039 & 0.701 & $45.3 \pm 12.4$ & $45.2 \pm 11.8$ & 0.014 & 0.822 \\
\hline Glycated hemoglobin (\%) & $6.0 \pm 0.9$ & $6.0 \pm 1.3$ & 0.020 & 0.834 & $6.0 \pm 0.9$ & $6.0 \pm 1.3$ & 0.018 & 0.779 \\
\hline Fasting blood sugar (mg/dL) & $127.8 \pm 45.7$ & $124.4 \pm 56.8$ & 0.065 & 0.507 & $125.3 \pm 43.2$ & $125.4 \pm 55.8$ & 0.003 & 0.960 \\
\hline Aspartate transaminase (IU/L) & $27.5 \pm 12.2$ & $28.1 \pm 11.8$ & 0.053 & 0.601 & $28.1 \pm 13.1$ & $27.8 \pm 11.4$ & 0.026 & 0.680 \\
\hline Alanine transaminase $(\mathrm{IU} / \mathrm{L})$ & $18.7 \pm 9.8$ & $20.1 \pm 11.2$ & 0.137 & 0.168 & $19.0 \pm 9.8$ & $19.7 \pm 10.9$ & 0.067 & 0.302 \\
\hline eGFR $\left(\mathrm{mL} / \mathrm{min} / 1.73 \mathrm{~m}^{2}\right)^{*}$ & $90.2 \pm 41.9$ & $92.3 \pm 49.7$ & 0.046 & 0.643 & $92.7 \pm 44.0$ & $92.0 \pm 50.7$ & 0.016 & 0.806 \\
\hline \multicolumn{9}{|l|}{ Baseline scores } \\
\hline Initial NIHSS & $10(3-15)$ & $9(2-13)$ & 0.155 & 0.110 & $10(3-14)$ & $9(3-13)$ & 0.037 & 0.570 \\
\hline Previous mRS & $0(0-3)$ & $0(0-2)$ & 0.119 & 0.391 & $0(0-3)$ & $0(0-2)$ & 0.033 & 0.605 \\
\hline $\mathrm{CHA}_{2} \mathrm{DS}_{2}$-VASc score & $5(4-6)$ & $5(4-6)$ & 0.122 & 0.182 & $5(4-6)$ & $5(4-6)$ & 0.002 & 0.978 \\
\hline HAS-BLED score & $3(2-3)$ & $3(2-3)$ & 0.075 & 0.292 & $3(2-3)$ & $3(2-3)$ & 0.003 & 0.958 \\
\hline \multicolumn{9}{|l|}{ Acute treatment } \\
\hline Intravenous alteplase & $38(29.2)$ & $100(28.3)$ & 0.020 & 0.935 & $141(29.4)$ & $138(28.6)$ & 0.017 & 0.822 \\
\hline Mechanical thrombectomy & $25(19.2)$ & $52(14.7)$ & 0.120 & 0.290 & $81(16.9)$ & $76(15.8)$ & 0.029 & 0.695 \\
\hline Statin & $64(49.2)$ & $195(55.2)$ & 0.120 & 0.284 & $261(54.6)$ & $261(54.0)$ & 0.011 & 0.912 \\
\hline \multicolumn{9}{|l|}{ Cerebral artery status } \\
\hline Severe stenosis & $19(14.6)$ & $26(7.4)$ & 0.233 & 0.024 & $43(9.0)$ & $44(9.1)$ & 0.003 & 1.000 \\
\hline Any cerebral atherosclerosis & $105(80.8)$ & $252(71.4)$ & 0.220 & 0.049 & $358(74.7)$ & 357 (73.9) & 0.020 & 0.826 \\
\hline \multicolumn{9}{|l|}{ CMB status } \\
\hline CMB burden & $2(1-3)$ & $2(1-3)$ & 0.005 & 0.963 & $2(1-3)$ & $2(1-3)$ & 0.017 & 0.789 \\
\hline Lobar CMB & $81(62.3)$ & $194(55.0)$ & 0.149 & 0.179 & $271(56.6)$ & 275 (56.9) & 0.006 & 0.962 \\
\hline
\end{tabular}

Values are presented as mean \pm standard deviation, number $(\%)$, of median (interquartile range).

IPTW, inverse probability of treatment weighting; OAC, oral anticoagulant; Std diff, standardized difference; TIA, transient ischemic attack; LDL-C, low-density lipoprotein cholesterol; HDL-C, high-density lipoprotein cholesterol; eGFR, estimated glomerular filtration rate; NIHSS, National Institutes of Health Stroke Scale; mRS, modified Rankin Scale; $\mathrm{CHA}_{2} \mathrm{DS}_{2}$-VASc, Congestive heart failure, Hypertension, Age over 75 years (doubled), Diabetes mellitus, Prior Stroke or TIA (doubled), Vascular disease, Age 65 to 74 years, Sex category; HAS-BLED, Hypertension, Abnormal renal and liver function, Stroke, Bleeding, Labile INR, Elderly, Drugs or alcohol; $\mathrm{CMB}$, cerebral microbleed.

${ }^{*}$ As calculated using the Modification of Diet in Renal Disease formula. 
Supplementary Table 2. Efficacy and safety of oral anticoagulant and antiplatelet therapies

\begin{tabular}{|c|c|c|c|c|c|}
\hline \multirow{2}{*}{ Independent variable } & \multicolumn{5}{|c|}{ Hazard ratio (95\% confidence interval) } \\
\hline & Stroke & Recurrent IS & $\mathrm{ICH}$ & All-cause death & MACE \\
\hline \multicolumn{6}{|l|}{ All patients } \\
\hline Antiplatelet therapy & Reference & Reference & Reference & Reference & Reference \\
\hline Anticoagulants & $0.42(0.23-0.76)$ & $0.39(0.20-0.76)$ & $0.72(0.24-2.15)$ & $0.71(0.42-1.22)$ & $0.46(0.27-0.80)$ \\
\hline VKA & $0.48(0.26-0.88)$ & $0.42(0.21-0.85)$ & $0.83(0.28-2.57)$ & $0.71(0.41-1.25)$ & $0.53(0.30-0.93)$ \\
\hline NOACs & $0.22(0.06-0.74)$ & $0.27(0.08-0.92)$ & $0.31(0.04-2.89)$ & $0.73(0.35-1.52)$ & $0.24(0.08-0.72)$ \\
\hline \multicolumn{6}{|c|}{ Patients with multiple CMBs } \\
\hline Antiplatelet therapy & Reference & Reference & Reference & Reference & Reference \\
\hline Anticoagulants & $0.38(0.19-0.75)$ & $0.37(0.17-0.82)$ & $0.49(0.16-1.50)$ & $0.49(0.26-0.95)$ & $0.38(0.20-0.74)$ \\
\hline VKA & $0.43(0.22-0.87)$ & $0.40(0.18-0.92)$ & $0.54(0.17-1.73)$ & $0.44(0.22-0.89)$ & $0.45(0.23-0.87)$ \\
\hline NOACs & $0.21(0.05-0.89)$ & $0.28(0.06-1.24)$ & $0.31(0.04-2.62)$ & $0.69(0.29-1.67)$ & $0.17(0.04-0.76)$ \\
\hline \multicolumn{6}{|c|}{ Patients with strictly lobar CMB } \\
\hline Antiplatelet therapy & Reference & Reference & Reference & Reference & Reference \\
\hline Anticoagulants & $0.36(0.17-0.75)$ & $0.37(0.16-0.89)$ & $0.50(0.16-1.59)$ & $0.96(0.46-1.97)$ & $0.51(0.25-1.01)$ \\
\hline VKA & $0.41(0.19-0.88)$ & $0.40(0.16-1.01)$ & $0.57(0.17-1.87)$ & $0.99(0.47-2.09)$ & $0.57(0.28-1.17)$ \\
\hline NOACs & $0.21(0.05-0.93)$ & $0.29(0.06-1.32)$ & $0.30(0.03-2.66)$ & $0.91(0.34-2.45)$ & $0.31(0.09-1.09)$ \\
\hline
\end{tabular}

IS, ischemic stroke; ICH, intracerebral hemorrhage; MACE, major adverse cardiovascular events; VKA, vitamin K antagonist; NOAC, non-vitamin K antagonist oral anticoagulant; $\mathrm{CMB}$, cerebral microbleed.

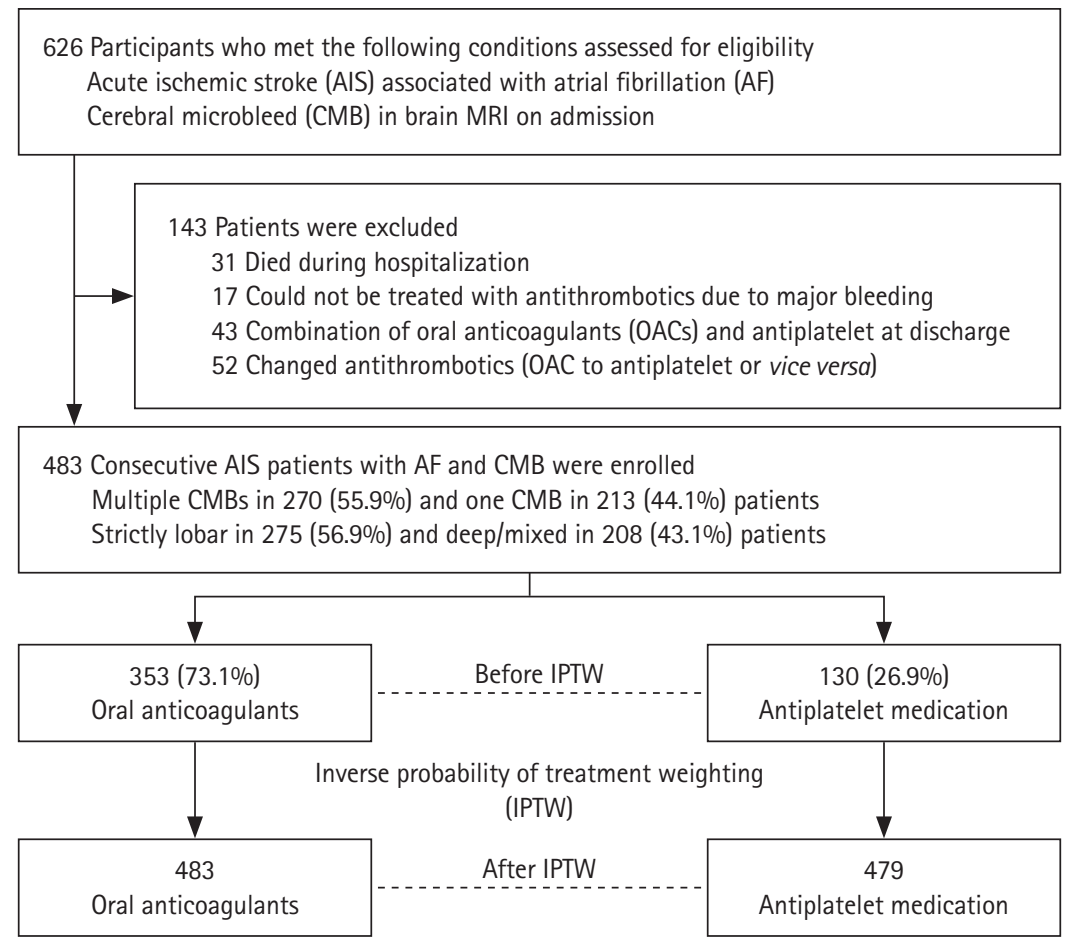

Supplementary Figure 1. Subject enrollment flowchart. MRI, magnetic resonance imaging. 


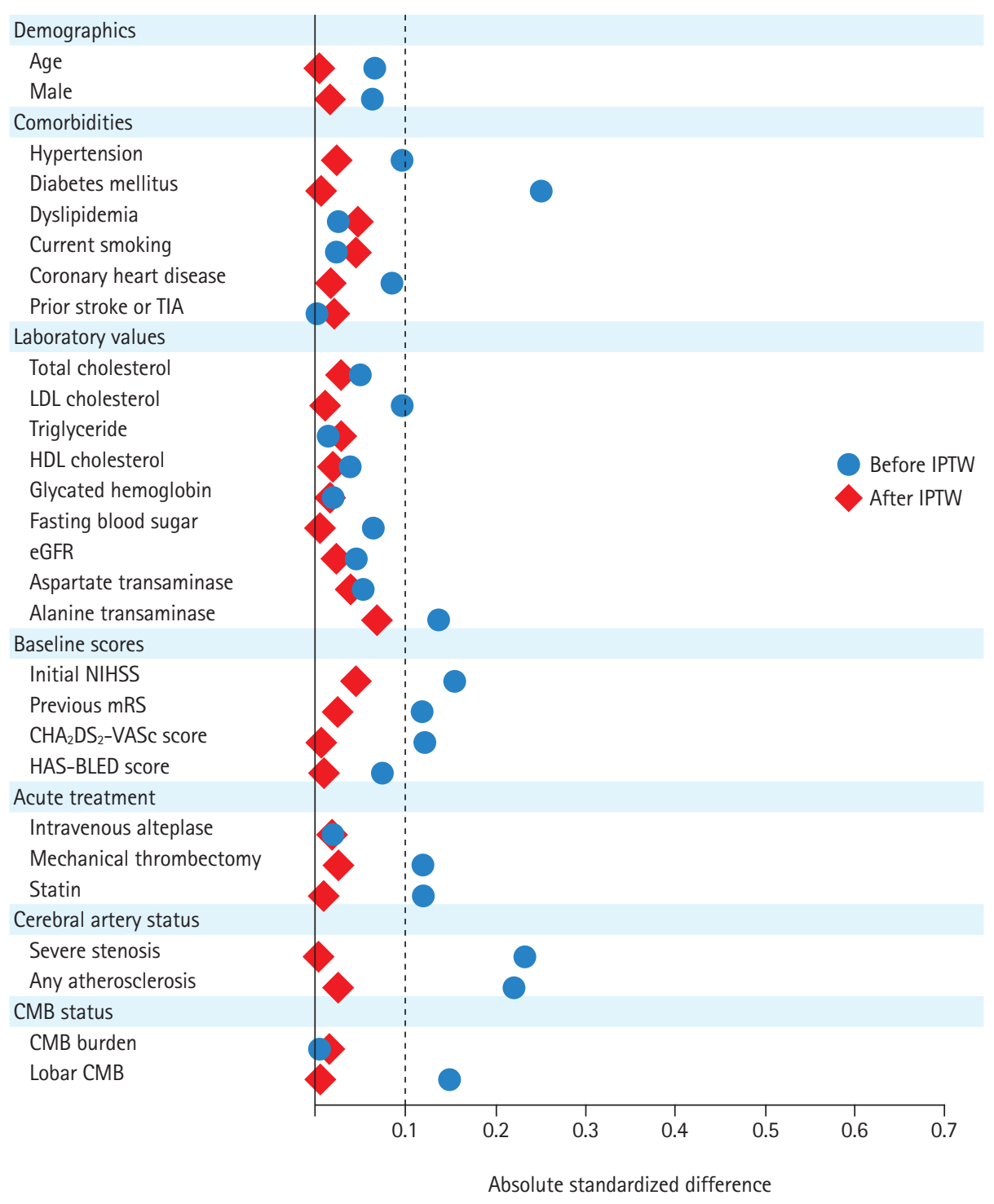

Supplementary Figure 2. Absolute standardized differences for estimating propensity score before and after inverse probability of treatment weighting (IPTW). TIA, transient ischemic attack; LDL, low-density lipoprotein; HDL, high-density lipoprotein; eGFR, estimated glomerular filtration rate; NIHSS, National Institutes of Health Stroke Scale; mRS, modified Rankin Scale; $\mathrm{CHA}_{2} \mathrm{DS}_{2}$-VASc, Congestive heart failure, Hypertension, Age over 75 years (doubled), Diabetes mellitus, Prior Stroke or TIA (doubled), Vascular disease, Age 65 to 74 years, Sex category; HAS-BLED, Hypertension, Abnormal renal and liver function, Stroke, Bleeding, Labile INR, Elderly, Drugs or alcohol; CMB, cerebral microbleed. 